\section{The Planck dive}

Phys. Rev. Lett. 109, 141103 (2012)

At the Planck scale, general relativity meets quantum mechanics. But what exactly takes place at this infinitesimal length scale $\left(10^{-35} \mathrm{~m}\right)$ remains largely speculative. Vahagn Gharibyan, however, suggests that there might be a way to test Planckscale effects using high-energy particleaccelerator experiments.

Theories of quantum gravity predict that — at the Planck scale — space-time has a crystal-like structure and refractive properties. Light from gamma-ray bursts and other astrophysical objects may display signatures of these quantum gravitational effects, but observations are limited by the uncertainty about the properties of these remote cosmological sources.

Gharibyan instead explores the possibility of glimpsing the effects of quantum-gravityinduced vacuum refractivity in Earth-based experiments: collisions between lasergenerated photons and relativistic electrons from current $\mathrm{GeV}$ and planned $\mathrm{TeV}$ particle accelerators. He estimates that, from a length scale of $10^{-31} \mathrm{~m}$ down to the Planck scale itself, subtle changes should manifest in measurable effects in high-energy Compton scattering.

\section{Lithium treatment}

Phys. Rev. Lett. 109, 141802 (2012)

In the standard model of particle physics there are three flavours of neutrino, and neutrinos are known to oscillate from one flavour to another. However, the field of neutrino research has thrown up certain data anomalies that could feasibly be explained in terms of the existence of a 'sterile' neutrino: one or more additional type of neutrino that doesn't take part in oscillations with its sibling particles.
Adriana Bungau and colleagues propose an accelerator-based experiment to address this issue. They envisage directing a $60 \mathrm{MeV}$ proton beam from a compact cyclotron onto a cylindrical beryllium- 9 target, to generate neutrons that are then thermally captured in a lithium-7 sleeve that surrounds the target. This creates lithium-8, which through beta decayat-rest generates a substantial flux of electron antineutrinos - sufficient, in combination with some large-scale scintillator detector (such as KamLAND or SNO+), to test the hypothesis of one or more sterile neutrinos.

The antineutrino source could be built within five years, say the authors, and could also be used to test other aspects of the standard model.

\section{Big science to go small}

Phys. Rev. X 2, 031019 (2012)

Phys. Rev. Lett. (in the press); preprint via

http://go.nature.com/Udw5Zi (2012)

Free-electron lasers generate high-intensity beams of coherent X-rays by manipulating the trajectory of relativistic electrons. But such sources are large and expensive. Two teams of researchers now present a blueprint for a compact source of coherent, long-wavelength radiation using a laser-plasma accelerator.

Laser-induced waves in a plasma can accelerate charged particles up to velocities near the speed of light. However, a beam generated in such a laser-plasma accelerator has not yet successfully seeded a free-electron laser, mainly due to the broad energy distribution of the accelerated electrons.

Andreas Maier and his colleagues have designed and modelled a two-metre long undulator - an arrangement of magnets that controls the direction of electron motion - that corrects for the energy spread by longitudinally decompressing the bunch of electrons. Similarly, Zhirong Huang

\section{Frosted icing} Proc. Natl Acad. Sci. USA 109, 16073-16078 (2012)

Striking though the formation of ice crystals may be, it is generally regarded as a hindrance in household and industrial settings alike. Extensive research has resulted in anti-ice surfaces that either repel or retard ice formation, but progress in their development is slowed by an incomplete understanding of the mechanism involved. Now, Stefan Jung and colleagues have determined a key role for evaporative cooling in the propagation of frost.

When a droplet attached to a surface freezes, it undergoes rapid crystal growth to form an ice-crystal scaffold. This structure is then filled out as the rest of the liquid freezes isothermally, releasing heat and inducing evaporation. It turns out that the resulting vapour condenses into a frost halo surrounding the droplet, as Jung et al. observed.

They found that the frost halo was capable of initiating freezing in neighbouring droplets effectively propagating frost formation across a surface. Using heat-transfer analysis and a diffusive vapour transport model, Jung et al. computed the size of the halo and used it to predict subsequent local freezing events. They found that halo size depends on the thermal conductivity of the substrate, adding another piece to the puzzle of how ice propagates. and co-workers considered an undulator that tackled the same problem, but acted instead to produce an optimized transverse field. Both undulator designs could work on electron beams with the energy-spread characteristics of state-of-the-art laserplasma accelerators.

\section{Behind the mask}

Nature 490, 514-516 (2012)

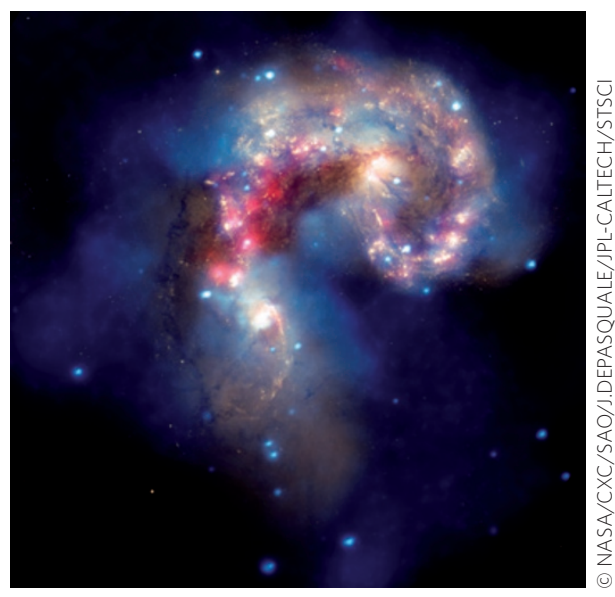

By using infrared space telescopes to measure the cosmic near-infrared background radiation, we are peering ever deeper into our past. Masking bright sources is one way of measuring this background, which is stronger than that expected from known galaxies. This excess radiation is thought to originate from cosmic sources created in the early Universe when massive stars and galaxies first formed, and from faint dwarf galaxies that are closer to Earth. Moreover, fluctuations in the brightness provide information on the number and distribution of the sources. Asantha Cooray and co-workers have analysed recent data taken by the Spitzer Deep, Wide-Field Survey, covering a vast 10.5 square degrees of the sky. They suggest an alternative infrared origin.

The authors confirm previous claims that, based on more spatially limited surveys, the fluctuations are ten times stronger than any radiation expected from primordial and dwarf galaxies combined. However, rather than seek new sources, Cooray et al. argue that the excess radiation arises from nearby stars within the dark-matter haloes that surround galaxies. These are stars that have been stripped from their parent galaxies during tumultuous galaxy mergers and collisions (pictured). The authors predict optical background light fluctuations with a similar clustering distribution.

Written by May Chiao, Iulia Georgescu, David Gevaux, Abigail Klopper and Alison Wright. 